\title{
16.4: The Optics of an Autostereoscopic Multiview Display
}

\author{
Eero Willman, H. Baghsiahi, F. A. Fernández, D. R. Selviah and S. E. Day \\ Dept. of $E$ \& E Eng., University College London, London, United Kingdom
}

\author{
Kishore V. C., E. Erden and H. Urey \\ Dept. of Electrical Engineering, Koç University. Istanbul, Turkey \\ P. A. Surman \\ Imaging \& Displays Research Group, De Montfort University, Leicester, United Kingdom
}

\begin{abstract}
An autostereoscopic head-tracked back projection display that uses an RGB laser illumination source and a fast light engine is described. Images are horizontally scanned columns controlled by a spatial light modulator that directs two or more images in the directions of the apposite viewers' eyes.
\end{abstract}

\section{Introduction}

The HELIUM3D (High Efficiency Laser-Based Multi-user Multimodal 3D Display) is a European Union funded project developing an auto-stereoscopic multiview multi-user display. The project is led by De Montfort University in the UK and comprises eight partners, the others being: Philips, Nanjing University, Barco, Fraunhofer HHI, Koç University, Technische Universiteit Eindhoven and University College London.

The goal of the project is the development of an auto-stereoscopic display whose capabilities exceed those of a purely stereoscopic display. The display operates in conjunction with a fast image forming device, allowing for the possibility of operating in what is referred to as the ' $3 \mathrm{D}+$ ' mode where a completely different image can be presented to each viewer's eye. This enables other interesting modes of operation to be achieved, for example; motion parallax, user-determined viewpoint, secure images visible only to selected viewer/s etc. A multi-user head position tracker is an integral part of the system and in addition to being used to locate the regions in the viewing field where the images are observed (exit pupils) the display lends itself readily to interactive applications where the knowledge of head positions is utilized.

\section{Operation of the HELIUM3D Display}

Figure 1 shows a schematic diagram of the HELIUM3D display system. The display consists of three sub-sections: the Light Engine, the Head Tracker and the Transfer Screen.

The Light Engine [1] is to deliver a vertical uniform white light column with the size of $8 \mathrm{~mm} \times 100 \mu \mathrm{m}$ which is scanned using a scanning mirror across a Liquid Crystal on Silicon (LCOS) light valve. Three high power multi-emitter lasers are employed for red $(640 \mathrm{~nm})$, green $(532 \mathrm{~nm})$ and blue $(465 \mathrm{~nm})$ to achieve a wide color gamut and deliver enough luminance intensity at the LCOS device. Using multi emitter lasers reduces the coherence length and helps in combating speckle, which is a real concern in laser projection systems. The initial power of each laser is $3 \mathrm{~W}$ and a color temperature of $6500^{\circ} \mathrm{K}$ is achieved by using a power ratio of 1: 0.49 : 0.63 for red, green and blue. The three lasers are combined, homogenized and shaped using an x-cube, optical lenses and microlens arrays, and delivered as a uniform light column at the position of the LCOS device. The LCOS contains the image content thus creating a horizontally scanned columnar representation of the image.
The scanned image is relayed to the transfer screen stage using a conventional multi-element projection lens $\left(\mathrm{L}_{1}\right)$. This lens acts as an interface between the Light Engine and the Transfer Screen sub-systems of the display.

The head tracker stage identifies the locations of the viewers' left and right eyes in real time [2]. The eye positions are used for controlling a ferroelectric liquid crystal spatial light modulator (SLM) within the transfer screen. The SLM steers the scanned image columns to the viewers' left or right eyes. The different modes of operation of the display are realized using time domain multiplexing of the displayed image frames.

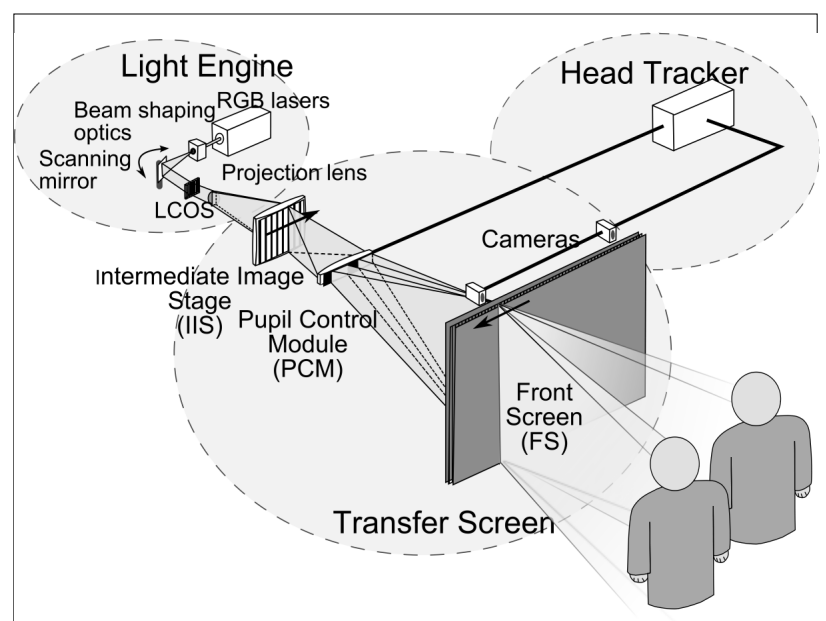

Figure 1. A simplified schematic diagram showing the subsystems comprising the HELIUM3D display.

\subsection{The Transfer Screen}

The transfer screen can further be sub-divided into three separate units: The intermediate image stage (IIS), the pupil control module (PCM) and the front screen (FS).

The IIS consists of a lens $\left(\mathrm{L}_{2}\right)$ followed by a horizontal diffuser. $\mathrm{L}_{2}$ acts as a field lens, redirecting the scanned image column towards the center of the PCM, while the horizontal diffuser spreads the beam to cover the whole width of it. The PCM contains a lens $\mathrm{L}_{3}$ and a high-speed linear ferroelectric liquid crystal SLM. $\mathrm{L}_{3}$ projects the image formed on the intermediate image stage onto the surface of the front screen. The SLM is made of a large number of narrow vertical pixels arranged side-by-side along the horizontal axis. The states of the pixels are controlled individually to form sections along the length of the SLM where it is either in transmission or extinction. These sections act as dynamically reconfigurable apertures whose sizes and positions 
are continuously updated during each image frame. The position of an open aperture introduces a small angular deviation, $\alpha$, to the angle at which an image is incident on the FS (see figure 2). This angle determines the location of the exit pupil.

The FS consists of four layers: $\mathrm{L}_{4}, \mathrm{G}_{1}, \mathrm{~L}_{5}$ and $\mathrm{V}_{1}$. Layers $\mathrm{L}_{4}$ and $\mathrm{L}_{5}$ are aspherical Fresnel lenses, $\mathrm{G}_{1}$ is a Gabor Superlens (see section 3) and $V_{1}$ is a vertical diffuser.

$\mathrm{L}_{4}$ is located at a distance equal to its focal length $\left(f_{4}\right)$ from the PCM, collimating the transmitted light, but leaving the angle $\alpha$ unaffected. After this, the superlens screen acts as a beam deflector with gain, magnifying $\alpha$ by a factor $M$. Lens $\mathrm{L}_{5}$ is a field lens that focuses the transmitted beams back toward the optic axis of the system. The focal length, $f_{5}$, of lens $\mathrm{L}_{5}$ determines the range of valid viewing distances (the shaded area in figure 2). A shorter focal length allows the viewers to be located closer to the display and vice versa. A conjugate plane at distance $f_{5}$ from the front screen can be identified. At this distance the locations of the open apertures on the SLM do not change during the scanning of a frame (assuming viewer movement is negligible during this time). Finally, the vertical diffuser $\mathrm{V}_{1}$ allows for the exit pupil to be located at any position along the vertical axis.

\section{Gabor Superlens Screen}

In 1940, D. Gabor described superlenses as collections of microlens arrays that together behave like optical systems much larger than the individual lenticules [3]. In general, the optical properties (e.g. focal length) of superlenses can be altered by combining microlens arrays of slightly differing pitches. For example, the imaging properties of superlenses are studied in [4]. However, in this work we concentrate on the special case of using cylindrical microlens arrays of equal pitch, but differing focal lengths.

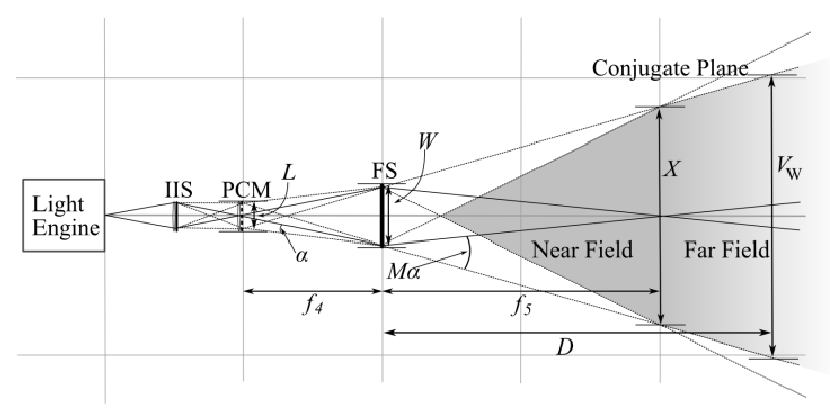

Figure 2. Top view showing the optics of the HELIUM3D display (approximately to scale).

Superlenses of equal pitch essentially work as beam deflectors and have been used in 3D display applications e.g. in $[5,6]$. The superlens screens described here additionally incorporate an element of gain or magnification; the screen magnifies the angle $\alpha$ by a factor $M$ in the horizontal plane, increasing the width of the viewing field. Various possible arrangements of microlens arrays for beam deflecting optical devices have been studied e.g. in [7]. Possible compositions include Keplerian and Galilean telescope arrangements. In the HELIUM3D display, a reversed Keplerian layout including a field lens array is used (see figure 3).

The superlens screen consists of three layers of cylindrical microlens arrays: An objective lens (layer 1), a field lens (layer 2) and an eyepiece lens (layer 3 ). As light enters the superlens, the first layer focuses the beam within the second layer where its direction is changed by the field lens. After this, the third layer collimates the transmitted light. A maximum allowed incident angle $\alpha_{\max }$ exists. When $|\alpha|$ is larger than this angle, the first point of focus is within an adjacent lenticule, resulting in the transmitted beam leaving the superlens in the incorrect direction. This occurs with a period of $\alpha_{\max }$.

In the case of a Keplerian telescope arrangement of lenses the magnification is the ratio of the focal lengths of the objective and the eyepiece lenses, $f_{1}$ and $f_{2}$ respectively. In the superlens, these are related to the thicknesses of the layers by $f_{1}=T_{1}+T_{2}$ and $f_{2}=T_{3}$.

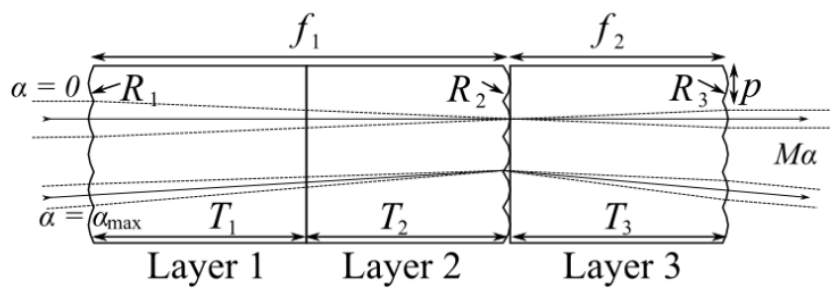

Figure 3. A section of a triple layered Gabor superlens. Ray bundles of collimated light are shown entering from the left at two different angles of incidence.

This means that in order to maximize the magnification, the third layer should be thin compared to the combined thickness of layers 1 and 2 . In practice, a thin third layer may become structurally unsound and pose restrictions on the manufacturing process of the screen.

\subsection{Superlens Screen Assembly Procedure}

Single sided cylindrical microlens arrays are readily available from many manufacturers. However, accurately aligned multilayered microlens arrays of the required thicknesses, focal lengths etc. are not as common. Instead, in this work, the Gabor superlens screens are assembled from single sided cylindrical microlens arrays. Correct alignment of the layers is vital to the performance of the final superlens screen. A three step procedure outlined below has been found to result in a uniform and accurate alignment:

1) Figure 4 (a). A low power laser is set on a horizontal translation stage. A one dimensional diffuser (e.g. a section of a cylindrical microlens array) is placed in the path of the beam in order to produce a narrow vertical beam. Vertical lines corresponding to different laser locations along the translation stage are marked on a target screen.

2) Figure 4 (b). Layers 1 and 2 are lightly clamped together in order to allow for relative movement. The relative positions of the screens are then adjusted so that the dimly lit regions on the target screen are optimized for being vertical and centered on the marked lines. Layers 1 and 2 are then securely fixed.

3) Figure 4 (c). Layer 3 is added to the assembly, again using light clamping. The position and orientation of layer 3 is then adjusted to align the resulting narrow vertical beams with the markings on the screen. Finally layers 1,2 and 3 are all securely fixed together

Layer misalignment can be detected by naked eye as Moire-like artifacts on the superlens screen. 


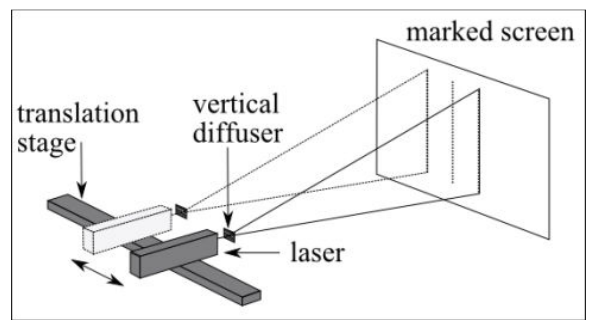

(a)

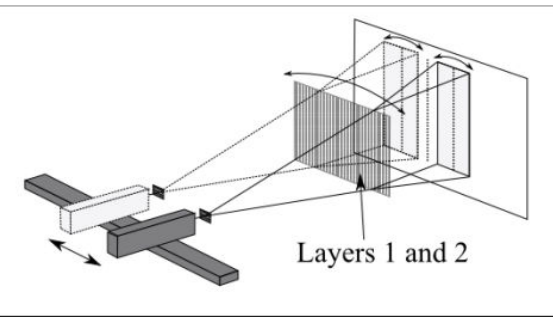

(b)

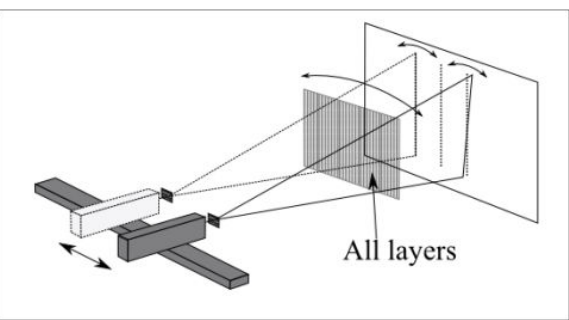

(c)

Figure 4. Three-step alignment procedure for assembling Gabor superlenses.

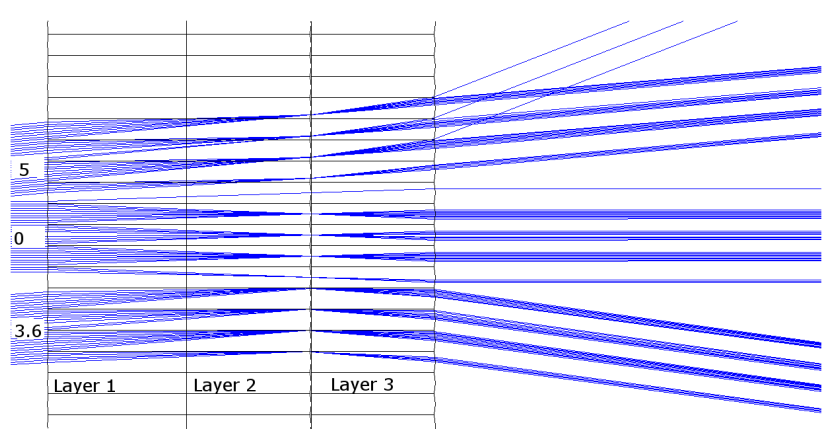

(a)

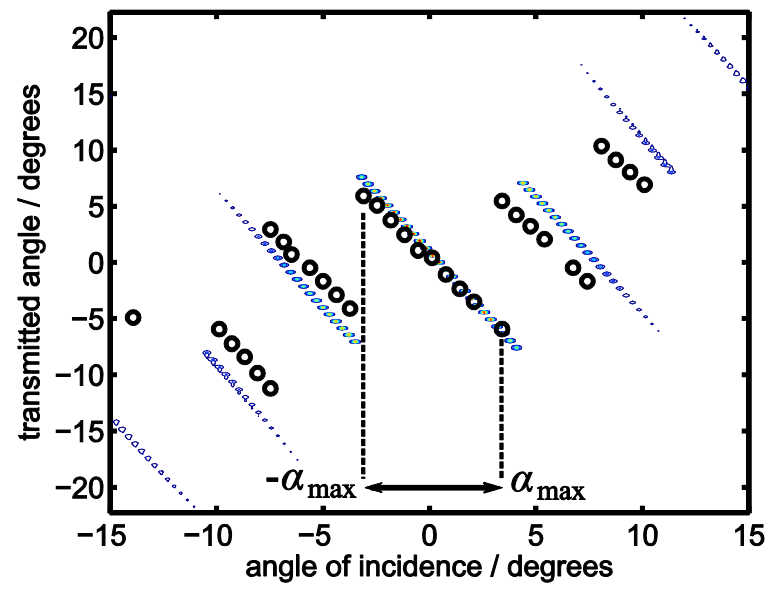

(b)

Figure 5. (a) Raytracing result showing the operation of the low magnification Gabor superlens screen. (b) Experimentally measured (bold black circles) and raytraced (thin colored line) transmission angle as a function of angle of incidence. The operation range of the superlens screen has been marked.

\subsection{An Initial Low Magnification Superlens Screen}

An initial triple layered superlens screen has been assembled using the procedure described above. The microlens arrays used in the initial screen are standard off-the-shelf components and the magnification of the screen is only around a factor of two. A higher magnification screen with custom designed high precision lenticulars with acylindrical surface profiles has been designed and is currently under construction.
The pitch of the lenticular screens is $0.564 \mathrm{~mm}$ and the refractive index of the material is 1.58. The other parameters are given in table 1. The maximum angle of incidence of the screen is approximately $\alpha_{\max } \approx \pm 3.7$ degrees. The operation of the initial screen is shown in figure 5 (a) by means of a ZEMAX raytrace simulation. Rays are shown entering from left at $\alpha=0,3.6$ and 5 degrees. Figure 5 (b) shows a comparison of the angle of incidence vs. transmitted angle between results obtained from experimental measurements and from ray trace simulations. The results show good agreement. The performance of the measured superlens screen is only slightly worse compared to that of the theoretical results; both the magnification and $\alpha_{\max }$ are slightly lower than predicted.

Table 1 Preliminary superlens screen parameters.

\begin{tabular}{|c|c|c|}
\hline & Radius / mm & Thickness / mm \\
\hline Layer 1 & 2.584 & 3.72 \\
\hline Layer 2 & 1.219 & 3.32 \\
\hline Layer 3 & 1.219 & 3.32 \\
\hline
\end{tabular}

\section{The Pupil Control Module}

The Pupil Control module comprises the spherical convex lens $\mathrm{L}_{3}$ and a linear SLM having $N_{\mathrm{A}}$ switchable vertical apertures. At a given position of the horizontal scan these aperture positions correspond to particular emergent horizontal angles from the screen. Although elongated, $\mathrm{L}_{3}$ is actually a spherical lens that is effectively the projection lens for the image on the Intermediate Image Stage.

The SLM must be fast in order to cope with exit pupils that are furthest from the conjugate plane of the SLM in the viewing field. This is due to the fact that the dynamic aperture forming the exit pupil must in general move position over the duration of the horizontal scan. The required refresh rate, $C$, of the SLM depends on many factors, including the position of the viewer, the focal lengths of the lenses in the front screen, the frame rate of displayed images and the speed of the light engine. A simple analysis of the required SLM response time is presented next.

\section{1 SLM Response Time Requirement}

The effective time duration of each image is $T R$, where $T$ is the time period of the frame and $R$ is the fraction of this time that the image is actually displayed for. Ideally $R$ equals one, but in reality, the image is not visible for the complete duration of the frame due to factors such as a finite response time of the LCOS unit and flyback time of the scanning mirror etc.

During this time, $T R$, the SLM is addressed $N_{\mathrm{A}} P$ times, where $N_{\mathrm{A}}$ is the number of sub-apertures and $P$ is a fraction indicating the 
amount of movement of the aperture opening with respect to the length $L$ of the SLM during a displayed image frame. This means that the required SLM refresh rate $C$ is given by:

$$
C=\frac{P N_{A}}{T R}
$$

The value of $P$ depends on the viewing distance $D$ and the optical properties of the front screen. It can be shown by considering similar triangles in the geometry shown in figure 2 that the width of the conjugate plane $X$ is given by:

$$
X=L M \frac{f_{5}}{f_{4}}
$$

and that the total width of the viewing field at distance $D$ from the front screen is:

$$
V_{w}=X \frac{D}{f_{s}}-W \frac{\left|D-f_{s}\right|}{f_{s}}
$$

Then, the proportion, $P$, of the width of the SLM that an open aperture section must travel during the scan of a single image frame is given by:

$$
P=\frac{W\left|D-f_{5}\right|}{D X}
$$

The effect of changing the value of the focal length $f_{5}$ of lens $\mathrm{L}_{5}$ in the front screen is shown in figure 6. The values for the other parameters used are given in table 2. In the limit of stationary viewers located at the conjugate plane and perfectly time-efficient light engine $\left(P=1 / N_{\mathrm{A}}, R=1\right)$, the required SLM speed equals that of the image refresh rate. A more realistic case allowing for full user movement $P=1$ and $R<1$, results in a significantly higher refresh rate. For example, assuming four viewers with $60 \mathrm{~Hz}$ refresh rate per eye, $N_{\mathrm{A}}=512$ and $R=0.7$ results in $C$ in the order of hundreds of $\mathrm{kHz}$. This can be realized using ferroelectric liquid crystal devices with microsecond switching times [8].

\section{Summary}

The operation of an autostereoscopic display being developed under the European Union Framework 7 program has been presented. In particular, the operation of the so-called front screen sub-section of the display and its optics has been described. The design and construction of a triple layered Gabor superlens screen has been discussed and an analysis of the response time requirement for a fast spatial light modulator has been presented.

Table 2. Parameter values used in example calculations.

\begin{tabular}{|c|c|}
\hline$f_{4}=762 \mathrm{~mm}$. & $L=150 \mathrm{~mm}$. \\
\hline$W=403 \mathrm{~mm}$. & $M=4$ \\
\hline
\end{tabular}

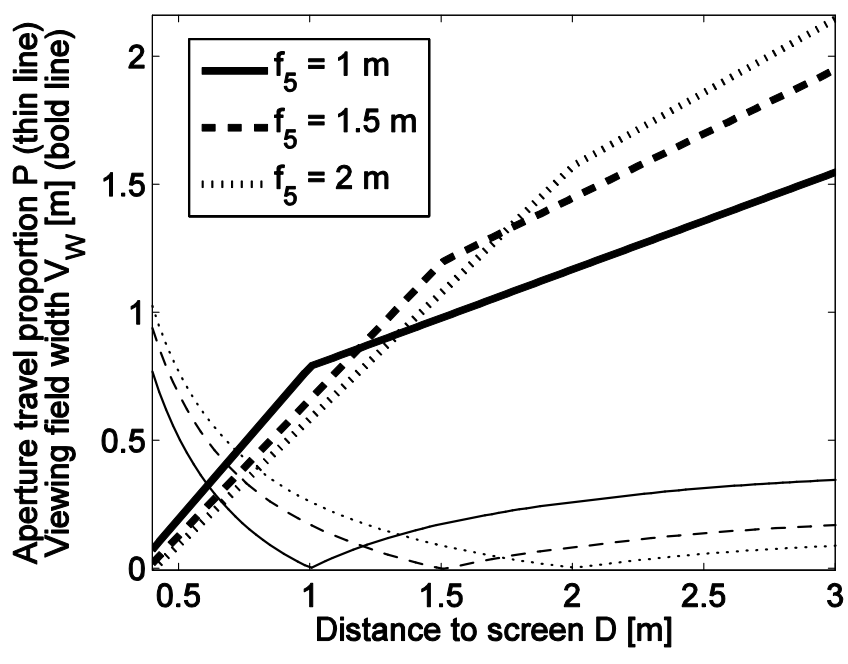

Figure 6. Plots showing the viewing field widths $V_{W}$ and the proportion $P$, of the SLM width that an aperture must travel during a single image frame as functions of the distance $D$ for three different focal lengths of lens $L_{5}$.

\section{Acknowledgements}

Funding from the European Union FP7 for the HELIUM3D project (contract No. 215280) is acknowledged. The authors would like to thank all the members of the HELIUM3D group.

\section{References}

[1] Kishore,V.C. et al,"Laser Scanning 3D Display with Dynamic Exit Pupil”, SID Eurodisplay, 492-495, (2009).

[2] Surman et al, "Head Tracked 3D Displays", Lecture Notes in Computer Science, 4105, 769-776, (2006).

[3] Gabor D, "Optical System Composed of Lenticules", UK Patent 541 753, (1940).

[4] C. Hembd-Sölner et al, "Imaging Properties of the Gabor Superlens”, J. Opt. A: Pure Appl. Opt. 1, (1999).

[5] N. Tetsukani et al, "A Study on a Stereoscopic Display System Employing Eye-position Tracking for Multi-viewers", SPIE Proceedings, "Stereoscopic Displays and Virtual Reality Systems", 2177, 135-142, (1994).

[6] K. Omura et al, Lenticular Autostereoscopic Display System: Multiple Images for Multiple Viewers", Journal of the SID, 4/6, 313-324, (1998).

[7] J. Duparré et al, "Implementation of Field Lens Arrays in Beam-Deflecting Microlens Array Telescopes", Appl. Opt., 43, 25, (2004).

[8] N. A. Clark, S. T. Lagerwall, "Submicrosecond Bistable Electro-Optic Switching in Liquid Crystals", Appl. Phys.Lett., 36, 899, (1980). 